\title{
Entre mar y tierra: desarrollo dual de las poblaciones prehispánicas del Manabí meridional, Ecuador
}

Entre mer et terre : développement dual des populations préhispaniques du Manabí méridional, Équateur

Between see and land: dual development of Prehispanic populations in South Manabi, Ecuador

\section{Yann Graber}

\section{OpenEdition}

Journals

Edición electrónica

URL: http://journals.openedition.org/bifea/1786

DOI: $10.4000 /$ bifea. 1786

ISSN: 2076-5827

Editor

Institut Français d'Études Andines

Edición impresa

Fecha de publicación: 1 diciembre 2010

Paginación: 603-621

ISSN: 0303-7495

Referencia electrónica

Yann Graber, «Entre mar y tierra: desarrollo dual de las poblaciones prehispánicas del Manabí meridional, Ecuador », Bulletin de l'Institut français d'études andines [En línea], 39 (3) | 2010, Publicado el 01 junio 2011, consultado el 09 diciembre 2020. URL : http://journals.openedition.org/bifea/1786 ; DOI : https://doi.org/10.4000/bifea.1786

\section{(c) (†) $\odot$}

Les contenus du Bulletin de l'Institut français d'études andines sont mis à disposition selon les termes de la licence Creative Commons Attribution - Pas d'Utilisation Commerciale - Pas de Modification 4.0 International. 


\title{
Entre mar y tierra: desarrollo dual de las poblaciones prehispánicas del Manabí meridional, Ecuador
}

\author{
Yann Graber*
}

\begin{abstract}
Resumen
La inestabilidad económica y política del Ecuador no ha favorecido la preservación del patrimonio cultural. Al contrario el huaqueo permitió incrementar los ingresos de algunas comunidades rurales. La prospección regional representaba efectivamente, en este caso, una buena alternativa para evaluar el potencial arqueológico, así como la realización de proyectos de desarrollo en relación, y la evolución de los patrones de asentamiento. Los resultados de 6 años de estudio (2002-2007), en los alrededores de Salango y Río Blanco, han puesto en evidencia una historia cultural prehispánica de más de cinco milenios, desde Valdivia II hasta el periodo Manteño, así como una densificación progresiva de la ocupación correlativa a la creciente complejidad de estas sociedades.
\end{abstract}

Palabras clave: Ecuador, Manabí, prospección, metodología, ocupación territorial

\section{Entre mer et terre : développement dual des populations préhispaniques du Manabí méridional, Équateur}

\section{Résumé}

L'instabilité économique et politique de l'Équateur n'a guère aidé les pouvoirs publics à préserver le patrimoine culturel. Par ailleurs, le pillage des sites archéologiques a constitué une source d'augmentation des revenus pour certaines communautés rurales. Aussi, la prospection régionale constitue-t-elle un moyen efficace d'évaluer le potentiel archéologique, l'évolution des modes d'occupation territoriale, ainsi que la réalisation de projets de développement en relation. Les résultats de 6 années d'étude (2002-2007), aux environs de Salango et Río Blanco, ont mis en valeur une

* Universidad de Neuchâtel, Espace Paul Vouga, CH-2068 Hauterive, Suiza. E-mail: yann.graber@ unine.ch 
histoire culturelle préhispanique de plus de cinq millénaires, du Valdivia Il à la période Manteño, ainsi qu'une densification progressive de l'occupation résultante de la complexification de ces sociétés.

Mots clés : Équateur, Manabí, prospection, méthodologie, occupation territoriale

\title{
Between see and land: dual development of Prehispanic populations in South Manabí, Ecuador
}

\begin{abstract}
In Ecuador, the chronic economic and political instability did not favour the preservation of cultural patrimony by the authorities and looting. In contrast, the latter did constitute a possible way of increasing the low income of some rural communities. Regional survey represents a good alternative in this case in order to evaluate the archaeological potential of a region, which could provide development alternatives, and the evolution of settlement patterns. The results of 6 years of study (2002-2007), near Salango and Río Blanco, show more than five millennia of Prehispanic cultural history, from Valdivia II until the Manteño Period, as well as a clear increase in the density of settlement through time in correlation with the increasing complexity of these societies.
\end{abstract}

Key words: Ecuador, Manabí, survey, methodology, settlement pattern

\section{INTRODUCCIÓN}

Los llamados «Cinco Cerros», frontera natural entre la provincia de Manabí y la recién creada de Santa Elena, constituyen una originalidad orográfica en el litoral ecuatoriano. La proximidad inmediata del océano y de los relieves de la Cordillera costanera origina un entorno muy húmedo, resultado de la condensación de las masas de aire marinas, que contrasta con la extrema sequía de la Península, al sur, y de la franja litoral entre Machalilla y el Cabo San Lorenzo. La erosión fluvial del Ayampe y de sus afluentes ha creado también una serie de valles de eje norte-sur separados físicamente del océano por cadenas bajas de lomas. De una superficie de $183 \mathrm{~km}^{2}$, el área de estudio seleccionado se extiende desde las estribaciones dominando la ciudad de Puerto López y el río Pital $\left(1^{\circ} 33^{\prime}\right.$ de latitud sur) hasta el río Ayampe ( $\left(1^{\circ} 40^{\prime}\right.$ de latitud sur) entre su desembocadura y el recinto de Guale. El límite oriental está marcado por el cerro Bola de Oro, cima más alta de la región que culmina a $800 \mathrm{msnm}\left(80^{\circ} 43^{\prime}\right.$ de longitud oeste). El año se divide entre dos estaciones relativamente bien marcadas: la una seca de junio a diciembre, meses de garúa, y la otra húmeda de enero a mayo. La pluviometría anual media oscila drásticamente sobre una microescala geográfica pasando de los $300 \mathrm{~mm}$ en la proximidad de la orilla pacífica a $1500 \mathrm{~mm}$ en las lomas. La topografía, la altitud, la exposición oceánica y la calidad de los suelos se combinan para crear un mosaico de formaciones vegetales. Una transición progresiva se observa desde el medio tropical seco litoral cubierto por una sabana con cactus candelabros y 
arbustos bajos hasta las fuertes pendientes de las elevaciones brumosas donde crecen especies altas (moral fino Chlorophora tinctoria, guayacán Tabebuia chrysantha, guarumo Cecropia litorales o cade Phytelephas aequatorialis, entre otras) cubiertas de epifitas tapando un denso sotobosque. La diversidad fáunica iguala la variedad vegetal con 69 especies de mamíferos registradas en el Parque Nacional Machalilla, reserva natural que se extiende al margen septentrional de nuestra área de prospección. El Pacífico entraña una riqueza marina poco común, recurso primordial en el desarrollo de las comunidades prehispánicas litorales. Salango sigue siendo uno de los puertos de descargue más importante de la costa ecuatoriana para la pesca de peces pelágicos pequeños. Fuera de la cabecera cantonal (Puerto López), el estudio abarca tres comunas políticas, es decir Salango, Las Tunas y Río Blanco al este.

\section{PROSPECCIONES PASADAS Y METODOLOGÍA}

Durante los últimos treinta años, la prospección arqueológica conoció un fuerte auge en relación, primordialmente, con nuevos enfoques metodológicos y consideraciones económicas. La costa ecuatoriana no fue ajena a este proceso y los proyectos, ante todo norteamericanos, se multiplicaron desde las intervenciones de la universidad de Columbia en la Península de Santa Elena (dirigida por Lanning en los años sesenta) o, una década después, de las academias de Illinois y de Calgary (conducidas por Lathrap y Raymond respectivamente) en Chanduy, en el curso medio del río Daule en la cuenca del Guayas o en el valle de Valdivia. El propósito era de poner en evidencia los procesos de asentamiento durante el periodo Formativo Temprano, o sea Valdivia. Los investigadores implicados multiplicaron sus trabajos consecutivamente en varias zonas litorales, implicándose e influenciando la nacida enseñanza de la Arqueología en el Ecuador. Coetánea a la investigación de Real Alto (valle de Chanduy), la Misión arqueológica española en el Ecuador integraba un componente regional abarcando el litoral esmeraldeño y el río Tiaone (Guinea, 1984).

Si el conocimiento del modo de vida se amplió y la secuencia cultural se afinó durante este lapso de tiempo, las modalidades de ocupación territorial, dependiendo extensamente de la metodología de campo empleada, no evolucionó de manera significativa. Las numerosas intervenciones ligadas a los grandes proyectos energéticos nacionales (producción petrolera, construcción de infraestructuras), fuera de añadir nuevos datos en esta problemática, usaron métodos de campo no apropiados, a nuestro parecer, para exponer las variables ligadas a la aprehensión de los modos de asentamiento prehispánicos. Se sumó una interdicción de publicación de los resultados, por parte de las compañías implicadas, felizmente abolida hace pocos años. Zeidler (1986; 1995; 2003; 2008; Zeidler \& Pearsall, 1994) llevó a cabo una profunda reflexión sobre los métodos de prospección regional en relación con su proyecto en el valle de Jama, Manabí central. Sobre una superficie de $765 \mathrm{~km}^{2}$, se registraron 239 sitios arqueológicos mayormente ubicados en los fondos de valle y en los alrededores de San Isidro, punto focal de la investigación al 
igual que Real Alto durante su primer acercamiento de los patrones de ocupación territorial (Zeidler, 1986). Las planicies aluviales, de extensión desconocida, fueron exploradas sistemáticamente con un reconocimiento pedestre y pocos sondeos. Mientras tanto, las áreas marginales se estudiaron por medio de un muestreo aleatorio estratificado resultando en la inspección de 125 cuadrículas de 1 hectárea, las cuales cubrían un porcentaje ínfimo de las superficies restantes, aunque estas se caracterizaban por un relieve marcado. Si Zeidler (1995) consideró que los métodos «tradicionales» de prospección estaban adaptados a las fases pioneras de la investigación arqueológica, tomando justamente el ejemplo de las observaciones de los cauces de ríos que utilizamos, y sus propuestas teóricas eran vigentes idealmente, varios sesgos en las premisas hipotéticas y obvios procesos tafonómicos se unían para relativizar las conclusiones de cada estudio elaborado en base a una metodología de muestreo. Guinea (1984) también advirtió, con razón, de los efectos erosivos y acumulativos que afectaban los barrancos y, correlativamente, su observación. Las variaciones de flujo de los ríos provocaban, a lo largo de los valles y con intensidad variable, la destrucción más o menos acelerada de las orillas y, consecutivamente, de los sitios arqueológicos yacentes en las terrazas aluviales. Bruscos cambios de cauce cortaron las antiguas ocupaciones y llevaron los vestigios río abajo. Guinea añadió que las crecidas depositaban material cultural en lugares donde nunca ocurrió una ocupación humana. Zeidler pudo prevalecerse por la primera aplicación a gran escala de un muestreo arqueológico en Ecuador, técnica aplicada actualmente por Alexander Martin (2009) en la región de Machalilla, añadiendo así un nuevo acercamiento en las numerosas intervenciones llevadas a cabo en el cantón de Puerto López.

Nacido de la iniciativa de Presley Norton, el Programa para la Antropología del Ecuador (PAE) propició una dinámica de investigación sin precedentes en el sur de la provincia de Manabí. Además de las excavaciones llevadas a cabo en la Isla de La Plata y, luego, en Salango entre los años 1979 y 1989, uno de los objetivos del programa era emprender amplias campañas de prospección arqueológica entre los ríos Buenavista y Ayampe, cubriendo más de $2000 \mathrm{~km}^{2}$. Varios investigadores unieron también sus esfuerzos en el reconocimiento del famoso Señorío de Salangome, como temática privilegiada de estudio, y las intervenciones se focalizaron sobre los importantes sitios manteños que lo conformaban como Agua Blanca (McEwan, 2003), Los Frailes (Mester, 1990) o Puerto López (Currie, 1995). Lamentablemente, el deseo declarado de una arqueología regional incorporando toda la secuencia cultural y la distribución ocupacional fue solamente esbozado dejando, como único testimonio, una maqueta tridimensional acribillada de alfileres multicolores indicando atribuciones crono culturales sin referencia 1 en el Centro de Investigación y Museo de Salango (CIMS). Al final de la década de los años 1970, Damp inició un reconocimiento de los ríos Pital (desembocando en Puerto López), Blanco y Ayampe. Durante este periodo, la región sufrió de una sequía extrema que permitió una buena visibilidad de superficie para la

1 Esta maqueta se conserva, de manera poco apropiada, encima de la biblioteca del dicho centro. 
localización de sitios arqueológicos. Sin embargo, los relieves seguían densamente cubiertos por el bosque. Damp, muy discreto en cuanto a su metodología de campo, pareció haber seguido las vías y caminos, considerándolos como sus transectos, hacia aldeas lejanas desde entonces abandonadas por la baja productividad agrícola, la caída de los precios y el éxodo rural. En Chanduy, como corresponsable del reconocimiento con Zeidler, escogió un método similar cuando mencionó haber realizado una prospección no probabilística siguiendo transectos accesibles adyacentes a los cauces. Dos documentos cartográficos resultaron de la prospección de Damp en la cercanía de Salango: un mapa elaborado bajo el auspicio del PAE2 seguramente en conjunto con McEwan, ya que constaban de los sitios arqueológicos de la zona de Agua Blanca y el plan de distribución de los asentamientos Valdivia en su artículo de 1984. En el primero figuraba toda la secuencia cultural regional mientras que el segundo resumía únicamente la ocupación del Formativo temprano, derivando del interés de Damp por este periodo desde su implicación en el proyecto de Real Alto dirigido por Lathrap. Usamos esta fuente de datos para ampliar nuestra muestra sobre todo desde el punto de vista topológico (el material cerámico conservado en Salango completó favorablemente los vestigios recolectados por nosotros lógicamente sesgado por la selección hecha en el campo) y, en menor grado, aumentó el inventario de sitios. Las otras intervenciones dentro del PAE quedan más informales. Consecutivamente a sus excavaciones en Río Chico (OMJPLP-170) y en Salango (OMJPLP-140), Allan \& Allan emprendieron, entre 1986 y 1987, una prospección sistemática de la desembocadura del río Salango y de las cercanías del pueblo actual. Escogieron una estrategia de pequeños sondeos distantes de $20 \mathrm{~m}$ sobre transectos predefinidos. Una disminución de la malla ( $5 \mathrm{~m}$ de distancia) era prevista durante una tercera campaña nunca efectuada. Allan \& Allan mencionan la presencia de, por lo menos, una estructura rectangular manteña y de reservorios dominando el asentamiento. La urbanización actual trasformó profundamente el paisaje y ningún cimiento de edificios prehispánicos era visible. Los acondicionamientos sugeridos en las lomas no fueron localizados durante nuestra prospección, tal vez completamente tapados por la densa vegetación local o por una intensa sedimentación. Al norte del pueblo y próximas al cauce del río, unas aldeas fueron registradas, que sugerían ocupaciones en el centro del valle por la presencia de material cultural. Se sospecharon, en este caso, fenómenos de deposición secundaria en esta zona sujeta a inundaciones, asemejándose entonces a las observaciones de Guinea (1984). Por su parte, Kreid registró 27 sitios en la isla Salango, concentración muy importante en comparación con la superficie restringida del islote. Sin rechazar los resultados de este reconocimiento que no concordaron con una exploración de la isla, estos revelaron la necesidad de una clara definición de la noción de sitio arqueológico.

En el presente proyecto, las redes hidrográficas regionales conformaron la base de la subdivisión zonal del área y cada campaña de prospección cubrió un promedio

2 Este documento se encuentra archivado en el Centro de Investigación y Museo de Salango. 
de $30 \mathrm{~km}^{2}$. El relieve pronunciado y la densa vegetación impusieron técnicas de campo oportunistas, las cuales incluían el reconocimiento de los cauces de ríos y de las zonas altas siguiendo caminos vecinales. El medio ambiente descrito y el tiempo de que se disponía para cada campaña de campo no permitieron la aplicación de transectos. La cobertura vegetal habría necesitado un esfuerzo colosal de desmonte al cual se sumaría la reticencia de ciertos dueños locales frente a tal intervención en su propiedad. El muestreo presentaba, de igual manera, ciertos sesgos. Si la estratificación biótica y el muestreo consecutivo (aleatorio o sistemático), como fase preliminar, se aplicaba en cualquier medio, factores regionales específicos influyeron drásticamente en la efectividad de los métodos de prospección ligados a esta metodología (prospección pedestre y microsondeos3) y ampliamente usados en el Ecuador. Así la hondura a la cual yacían numerosos vestigios impidió su hallazgo. La observación de los barrancos de río, obviamente afectados por la erosión fluvial, proveía no obstante la visión en profundidad deseada (Graber, 2008). El piso de vivienda Valdivia temprano excavado en Colimes, al borde del río Daule, y cubierto por 7,5 m de aluviones (Raymond, 2003), se reveló un ejemplo perfecto de estructuras inalcanzables con la técnica del muestreo. La prospección de cauces tenía la ventaja de actuar en la zona donde la frecuencia de asentamientos pasados era la más elevada, como lo expresó además Zeidler (1995), pero igualmente la más afectada por la actividad fluvial. Entre un fenómeno natural irremediable, una tafonomía caprichosa y su busca ideal de exhaustividad, el arqueólogo tuvo que conformarse con sesgos inevitables. La región de Salango-Río Blanco se prestó a la táctica oportunista llevada a cabo a lo largo del transcurso de nuestra prospección. Fuera de la ubicación rápida de los sitios a partir de los cauces de río, el relieve pronunciado proporcionó una visión relativa de la extensión mayor de las ocupaciones. En efecto, la proximidad de las pendientes impuso límites obvios a las áreas favorables a la instalación de las comunidades prehispánicas.

\section{LAS OCUPACIONES FORMATIVAS (3300-800 a. C.)}

Los modelos de ocupación territorial Valdivia propuestos hasta la actualidad derivan de los proyectos, y de la visión correlativa, llevados a cabo en el suroeste de la Costa ecuatoriana (Península de Santa Elena y provincia del Guayas) particularmente durante la década de 1970 (Damp, 1984; Schwartz \& Raymond, 1996; Zeidler, 1986; 1994). Basados en una metodología de muestreo, práctica fuertemente defendida por la Nueva Arqueología norteamericana desde sus inicios (Binford, 1964) en un intento de racionalización de la disciplina, ni la representatividad de los conjuntos considerados, ni las restituciones subsecuentes han sido evaluadas. Además se trasladan los patrones de repartición y de jerarquía de sitios a regiones distantes, con medio ambiente distinto, basándose en la idea de que la pertenencia a un horizonte cultural soluciona la apropiación territorial. Sin embargo, el

3 Los sondeos efectuados en el proyecto Jama alcanzaban apenas 40x40x40 cm (Zeidler, 1995) y muy pocas intervenciones usaban de cateos mayores. 
entorno influye drásticamente en los comportamientos y la organización de las comunidades prehispánicas, imponiendo respuestas socioculturales divergentes a pesar de recurrencias como en el aprovechamiento de las tierras cultivables, situadas primordialmente en los fondos de valles bordeando los cauces, por parte de sociedades básicamente agrícolas. Efectivamente, se privilegian las planicies aluviales en la ubicación de las comunidades y la mayoría de los 38 sitios Valdivia de nuestra área de estudio se alinean a lo largo de la franja costera o del valle del río Blanco (fig. 1). Una reevaluación del material y nuevos descubrimientos permiten reconsiderar el inicio de la ocupación Valdivia en su fase II (33002800 B.C.; Zeidler, 2003) y confirmar las aseveraciones de J. Damp (1984). Si los vestigios de esta época permanecen escasos (9 sitios), un incremento drástico de la cobertura ocupacional ocurre a partir del Valdivia medio con una triplicación del número de asentamientos. Las comunidades «pioneras» siguen su desarrollo, pero aldeas marginales, sin duda resultantes de las primeras, aparecen en los valles adyacentes o se alejaban del litoral oceánico. Durante el Valdivia final se observa una ligera disminución ocupacional (3 sitios menos). Estilísticamente, la cerámica de la región de Salango (fig. 2 A-C; G-I) se integra perfectamente a la secuencia tipo-cronológica de Hill (1972-1974) propuesta para la Península de Santa Elena y confirmada en el sitio de Real Alto (Marcos, 1988); para el Valdivia medio y tardío, tiene paralelos hasta en el alto Guayas (Guillaume-Gentil, 2008).

La variedad de medio ambientes propiciados por la cercanía del océano y de los relieves circundantes han favorecido la implantación de aldeas en tres zonas. Los dos sitios de playa registrados hasta ahora (Salango y Río Chico) se ubican en el margen de manglares, medio ambiente rico en recursos variados (conchas tales como Anadora tuberculosa, cangrejos o combustible). Apartados del litoral, a una distancia máxima de 1 kilómetro, asentamientos mayores ocupan las amplias terrazas aluviales de los valles bajos donde los campos se enriquecen periódicamente de sedimentos dejados durante las crecidas invernales. Un escenario idéntico caracteriza el valle del río Blanco. Fuera de un asentamiento Valdivia ubicado sobre un promontorio, las zonas bajas de la confluencia AyampeBlanco no parecen haber soportado aldeas antes del periodo Machalilla, pero su uso con fines agrícolas es muy probable. Los dos sitios de altura (al extremo norte de la zona de estudio y sobre las estribaciones occidentales del valle del Blanco) pertenecen a etapas relativamente tardías en la ocupación Valdivia y no permanecen sobre un largo periodo de tiempo, tal vez a favor de un periodo climáticamente favorable. Sin embargo, estos asentamientos se revelan excepciones al patrón de tierras bajas descrito previamente.

Si la continuidad cultural entre Valdivia y Machalilla se comprueba (Lippi, 1983; Staller, 2001), un hiato, de duración variable, persiste a lo largo de la costa meridional del Ecuador. Desde una posición cronológica flotante en la Península de Santa Elena (Paulsen \& Mc Dougle, 1974) hasta un claro abandono regional en el valle de Jama (Zeidler \& Pearsall, 1994; Zeidler, 2003) o en las provincias de Los Ríos y Cotopaxi (Guillaume-Gentil, en este volumen), la transición real y su foco permanecen desconocidos. En Jama, factores eruptivos influencian drásticamente las modalidades de ocupación territorial. Nuestra área de estudio, ubicándose al 


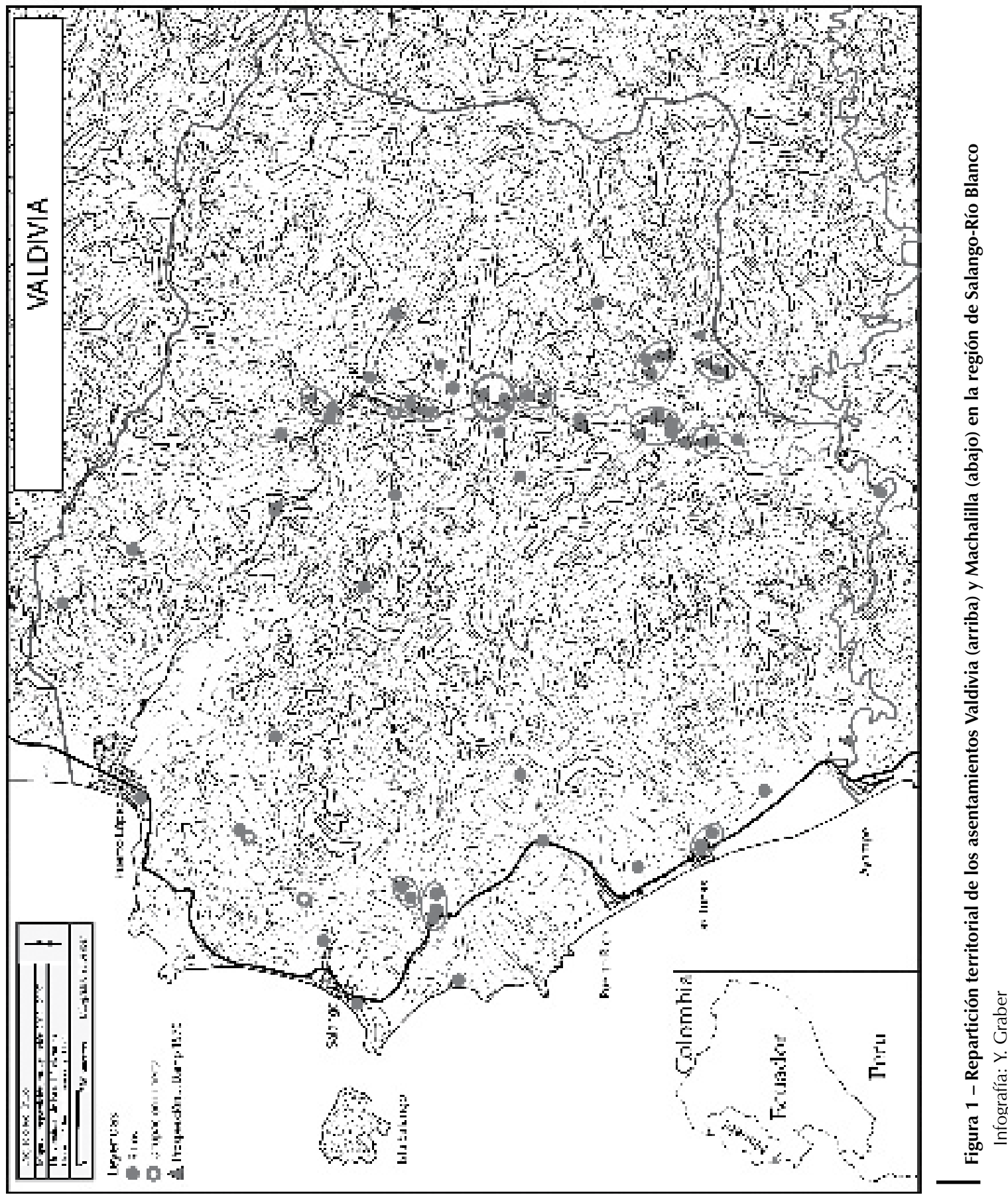




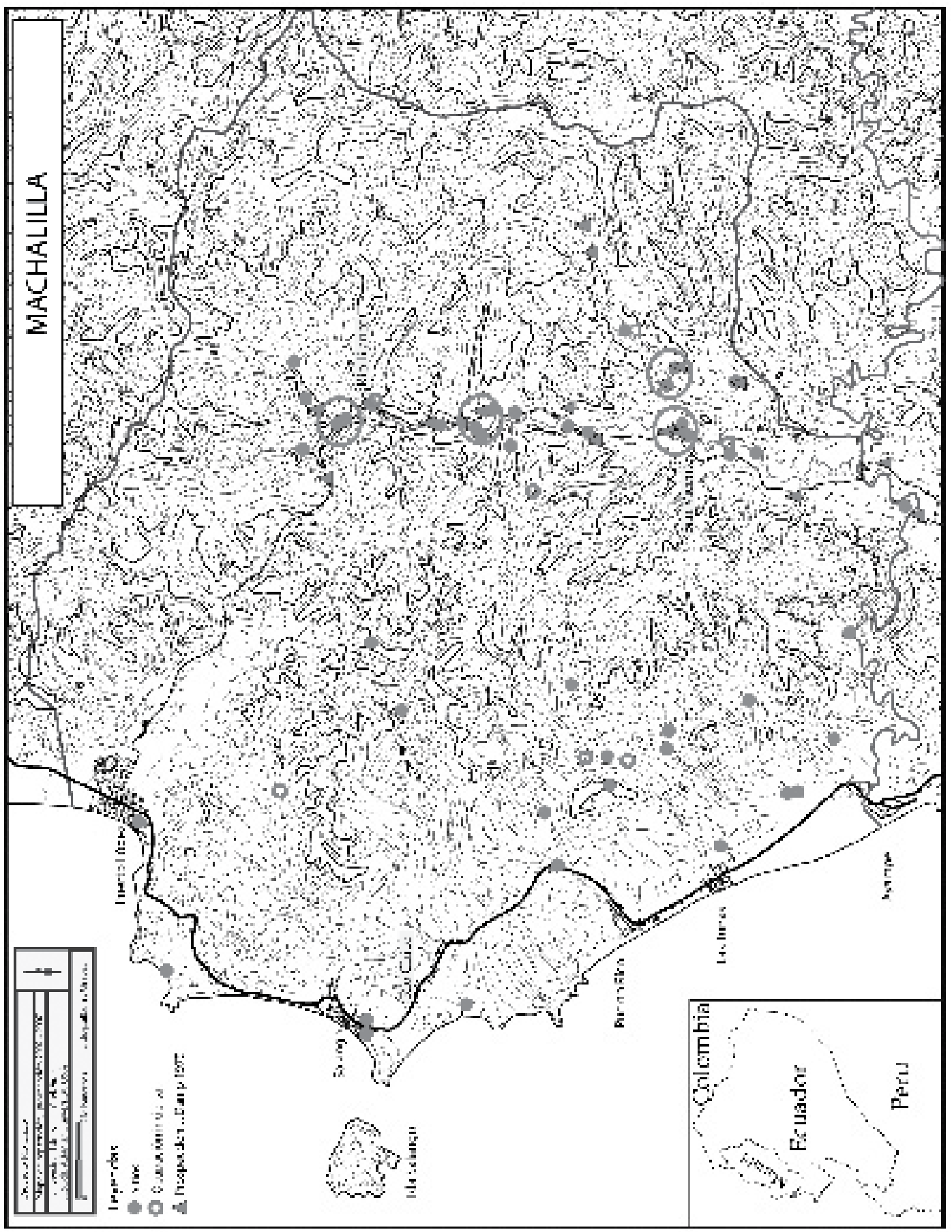




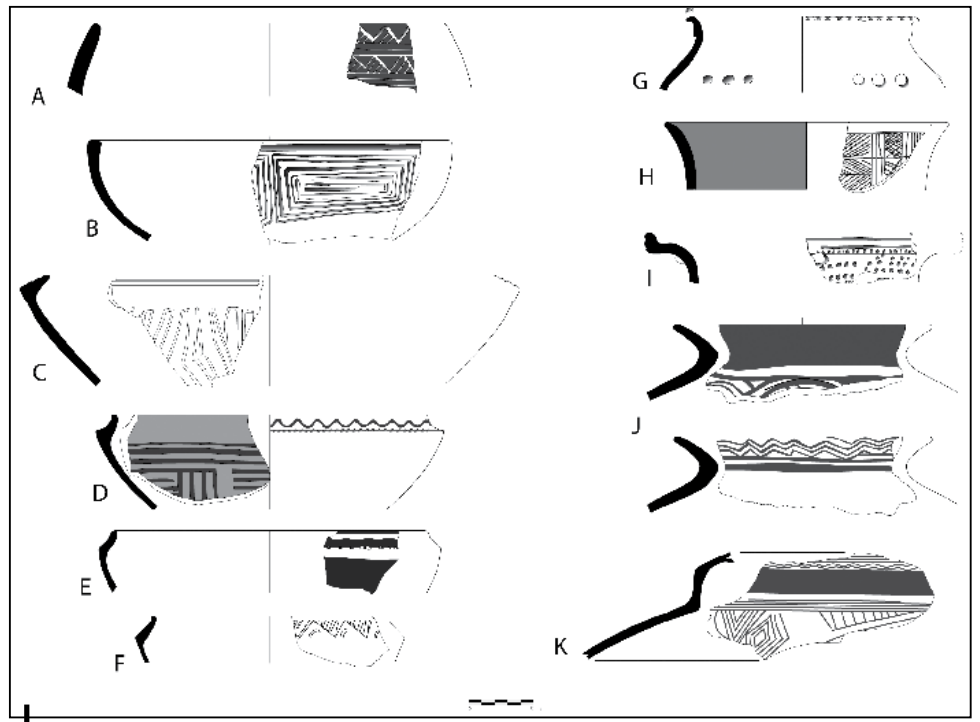

Figura 2 - Muestra cerámica Valdivia (A-C; G-I) y Machalilla (D-F; J-K) Infografía: Y. Graber

límite suroeste de la zona impactada por las caídas de cenizas volcánicas, padece igualmente estos fenómenos recurrentes, al contrario de lo avanzado por Zeidler (2003: 79)4, pero la recuperación poseruptiva puede haber sido rápida. Además otras causas, naturales o metodológicas, modelan la percepción actual de los procesos diacrónicos de ocupación territorial prehispánica y sus interrupciones efectivas o sugeridas. Lippi (1983), en base a su secuencia morfoestilística de los sitios La Ponga y Río Perdido, y observando rápidamente el componente Machalilla de las excavaciones de Salango, supone un inicio de esta ocupación, en el sur de Manabí, durante su fase La Ponga 4 (alrededor de 1100 a. C., fecha no calibrada). Su intuición evolucionista de la cultura material desde Valdivia final hasta Engoroy ofrece un eje de reflexión todavía vigente del cual podemos inferir, sin embargo, un principio ligeramente más temprano, en nuestra zona de estudio, es decir durante su fase La Ponga 3 (cerca de 1050 a. C., fecha no calibrada). Además, menciona la ausencia de los grandes cuencos ligeramente carenados Valdivia final en el sitio de La Ponga, aunque aparecen en su conjunto cerámico de Río Perdido en la Península y en el material recolectado en la región de Salango. Una cronología final falta todavía para esclarecer definitivamente la existencia real del hiatus mencionado o si, en ciertas regiones de la Costa e hipotéticamente, como en el sur de Manabí, la tradición Valdivia perdura algunos siglos mientras innovaciones Machalilla aparecen en zonas sureñas. La dispersión de las aldeas

4 Las correlaciones estrictas de los diferentes estratos de tefra evidenciados durante nuestra prospección necesitará unos análisis químicos ulteriores, pero la posición estratigráfica de estos depósitos, frente a niveles ocupacionales fechados por el material cultural, nos brinda una estimación sensata, el primero ocurre durante el Formativo final. 
Machalilla se revela muy similar tierra adentro (Río Blanco) en comparación con el periodo anterior, sugiriendo una perennidad de las modalidades ocupacionales y posiblemente una sucesión directa de las ocupaciones (fig. 1). Cerca de Las Tunas y en el valle del río Chico, se observa una expansión del aprovechamiento territorial hacia los valles litorales altos, mientras que un fenómeno inverso (concentración litoral) parece ocurrir más al norte. Damp, tampoco registra ocupaciones Machalilla en la zona del río Pital.

Schwartz \& Raymond (1996) observan, en el valle de Valdivia, un fenómeno de concentración poblacional a partir del Valdivia final, particularmente evidente durante el periodo Machalilla y que culmina en el Engoroy temprano. El esparcimiento aldeano —iniciado durante el Machalilla - se restringe y la población parece agruparse de manera privilegiada en la porción central del valle. En la zona de Salango y de Río Blanco, una persistencia ocupacional obvia de ciertos asentamientos importantes, ocupados desde el inicio del periodo Valdivia en la región, se percibe particularmente tierra adentro. No obstante, el número de sitios padece una drástica disminución durante el periodo Engoroy, reflejando la tendencia evidenciada en el valle de Valdivia o debido a factores cuya causalidad todavía está por establecer. Casualidad tal vez reveladora, el centro ceremonial de Salango inicia su auge a partir del siglo sexto antes de nuestra era, entonces durante el Engoroy medio (Lunniss, 2001; 2007; 2008) y, sin dudar que las actividades rituales han sido importantes anteriormente, se vuelve un lugar focal extrarregional por casi un milenio antes de su ocaso. En el valle de Jama, San Isidro sigue siendo, durante la fase Tabuchila (facies chorreroide local), el centro de la ocupación territorial. La mayoría de los asentamientos se ubican entonces en el fondo de los principales valles, sobre planicies aluviales, o a lo largo de los tributarios secundarios cercanos. Sin embargo, Zeidler (2008) sugiere la presencia de sitios en posición topográfica elevada. Se trata, a nuestro parecer, de aldeas situadas en los cursos altos de tributarios y/o de instalaciones dedicadas al aprovechamiento de ciertos recursos pero no de ocupación permanente. Más sorprendente es la falta completa de evidencias reportada por Zeidler (2003, 2008) en la orilla oceánica debida sin duda a la presencia moderna de piscinas camaroneras o, tal vez, a la metodología empleada. Parece poco probable que las comunidades prehispánicas hayan preferido, en esta zona, específicamente las áreas tierra adentro, en contraste con la totalidad del litoral ecuatoriano donde las aldeas ubicadas en las márgenes oceánicas están floreciendo. Una clara continuidad se observa en la franja litoral Puerto López-Ayampe en la selección de los lugares de establecimiento, es decir las playas de Puerto López, Playita, Salango y Río Chico, así como la desembocadura del río La Curia, al sur del actual pueblo de Las Tunas. Fuera del centro ceremonial de Salango cuyas actividades rituales se demarcan del resto de las aldeas, ciertos puntos focales emergen (Puerto López, Río Blanco y San Vicente) y tienen un desarrollo ininterrumpido durante los periodos posteriores. Al contrario, un fenómeno de abandono parcial parece ocurrir en las porciones altas de los valles (donde algunos sitios Machalilla han sido registrados) como si un acercamiento al océano se vuelve indispensable. 


\section{HACIA UNA DENSIFICACIÓN DE LA RED POBLACIONAL (100 a. C.-600/700 d. C.)}

El panorama de la ocupación territorial cambia totalmente durante el periodo Guangala (fig. 3). Los principales sitios del litoral o del valle del Blanco (Puerto López, Salango, Río Chico, Las Tunas, Río Blanco y San Vicente) perpetúan su dinámica interna de desarrollo y hasta amplían su superficie. La zona del actual pueblo de Río Chico es particularmente reveladora. A los escasos vestigios Engoroy sugiriendo un patrón disperso de viviendas o de grupos de habitaciones se sustituye un amplio asentamiento cubriendo decenas de hectáreas. En los cortes estratigráficos (8 y 15 m respectivamente) efectuados en el eje del poliducto MantaLa Libertad, se observa una secuencia de tres fases de construcción marcando la transición del Guangala medio a final hasta el inicio del periodo Manteño. Por el tamaño de los postes (más de $30 \mathrm{~cm}$ de diámetro), las habitaciones han debido alcanzar dimensiones importantes. Varios fogones se reparten tanto dentro de las

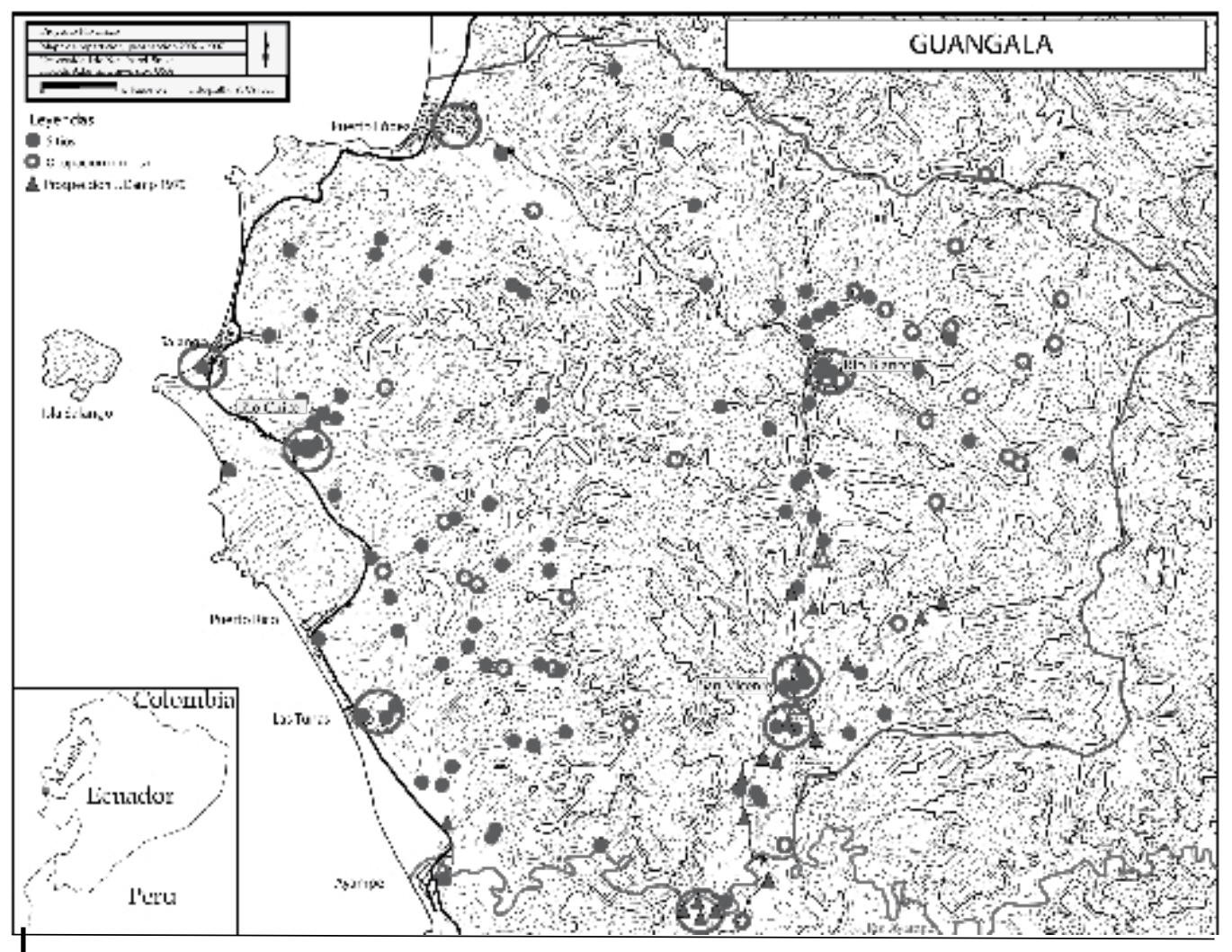

Figura 3 - Repartición territorial de los asentamientos Guangala (arriba) y Manteño (pág. siguiente) en la región de Salango-Río Blanco

Infografía: Y. Graber 


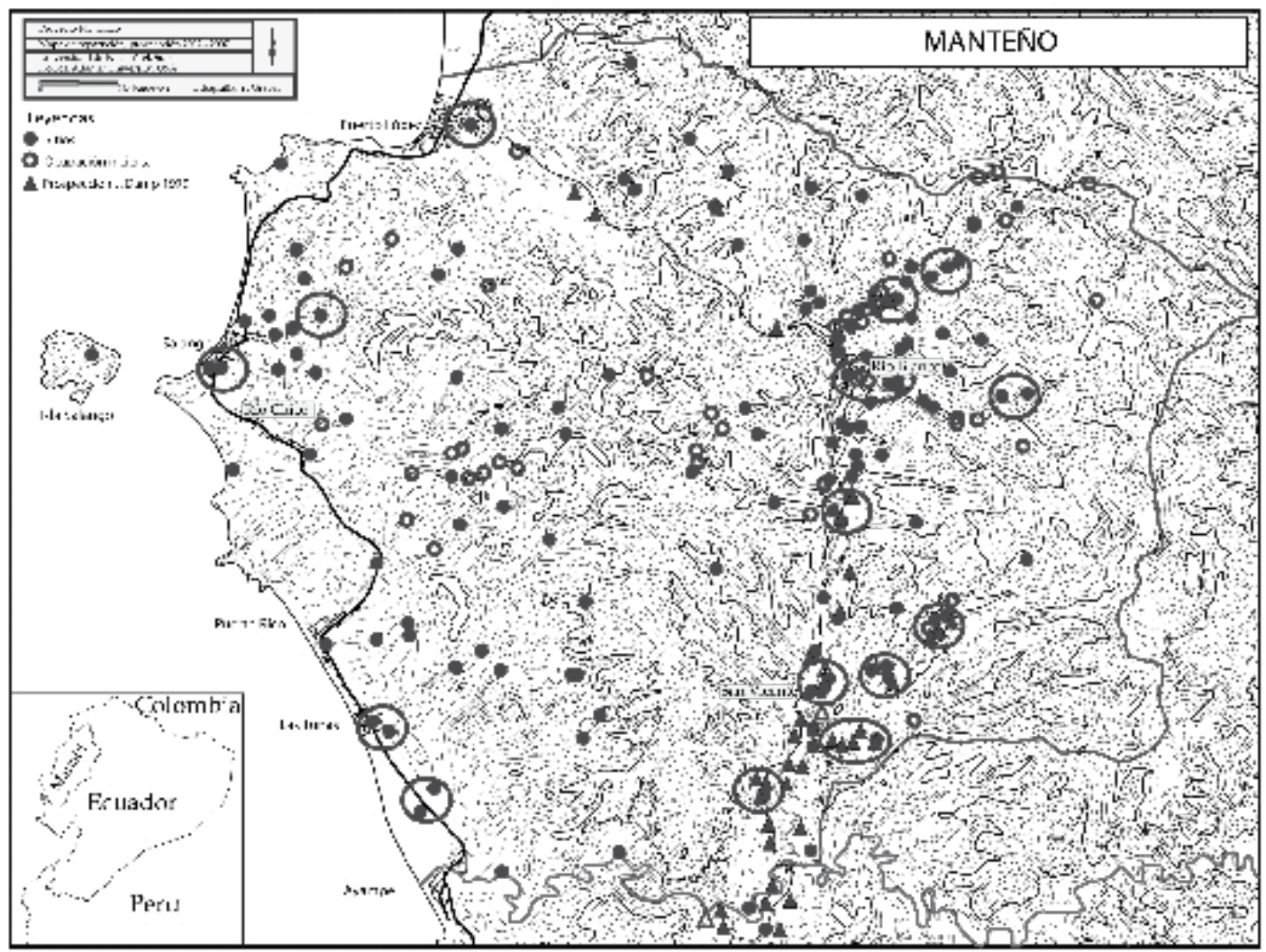

estructuras como fuera, distinguiendo tal vez actividades específicas. La intensidad de la ocupación se deduce también del imponente vertedero que se extiende $150 \mathrm{~m}$ (distancia observada) a lo largo del río Chico y tiene un espesor de 0,60 a $1 \mathrm{~m}$. Los recursos marinos (el océano está a menos de 2 kilómetros) destacan por su abundancia incluyendo conchas de manglar (Anadora tuberculosa) en gran cantidad, bivalvos y gasterópodos oceánicos (con una proporción no despreciable de Spondylus sp. y Pinctada mazatlanica), así como restos ictiológicos variados. Indicios de caza están igualmente representados y atestiguan el aprovechamiento de la integridad de los biotopos presentes, siendo el pueblo idealmente ubicado. Paralelamente, una profusión de pequeñas aldeas se dispersan en los valles litorales, reocupando generalmente lugares previamente abandonados, e intercalándose entre los pueblos principales del valle del río Blanco.

Durante los seis primeros siglos de nuestra era, el centro ceremonial de Salango cuenta con 10 episodios de remodelaciones (Lunniss, 2001) y perpetúa su influencia extrarregional. Creemos que el estatuto especial de Salango indujo una cierta confusión frente a la sucesión cultural regional. Norton et al. (1983) suponen una intensificación de la industria de transformación de los Spondylus y ostras mientras que el asentamiento llegaba al tamaño de: 
«una pequeña urbe con calles bien trazadas, plazoletas, sistemas de drenaje, edificios, recintos ceremoniales y cementerios (Norton et al., 1983: 54)».

Excepto una mención parecida a las crónicas de la Conquista, las cuales mencionan poblaciones semejantes provocando en cierto modo un anacronismo, la superposición ocupacional Bahía-Guangala está avanzada en base a dos fechas radiocarbónicas de contextos cerrados induciendo una posterioridad (50 a 230 años) de la última cultura (Norton et al., 1983: 61). En el mismo párrafo, se habla de un entierro que contiene vasijas de ambos estilos como depósito funerario. A un nivel regional, la secuencia Engoroy-Bahía-Guangala no se ha averiguado. Presentando en los sitios una clara transición, el material diagnóstico se asemeja a los conjuntos de la Península, entonces de afiliación Guangala. Esta conclusión no contradice el carácter mixto de la ocupación de Salango por la peculiaridad excepcional del sitio.

La obvia densificación de la red ocupacional durante el periodo Guangala (con más de 70 sitios registrados) no tiene, a nuestro parecer, paralelos conocidos actualmente en las regiones adyacentes aunque razonablemente el proceso debe reproducirse a gran escala. Una mención de Zeidler \& Pearsall (1994) sugiere, sin embargo, que este fenómeno de expansión territorial ha podido posponerse en ciertas regiones como en el valle de Jama:

«... la dispersión de asentamientos a través de áreas no-aluviales de valle [refiriéndose sin duda a zonas río arriba pero de fondo de valle: ndlr] no parece haber sido significativa hasta tiempos Jama-Coaque II después de 400 d. C. inmediatamente después del tercer evento volcánico que cubrió la región con otro nivel de tetra».

Esta erupción, atribuida hipotéticamente al volcán Atacazo y fechada entre 300500 de nuestra era (Isaacson, 1994), ha afectado igualmente, aunque con un impacto menor, el sur de Manabí. La distancia del litoral implica su alcance por eventos de magnitud extrema. Guillaume-Gentil (2008), en la región de La Maná al pie de los Andes, registra 9 niveles volcánicos puntuando su secuencia ocupacional desde el Pleistoceno Final. La tefra II de La Maná ocurre durante el tercer siglo de nuestra era: su contemporaneidad con la última capa de ceniza de Jama es altamente probable. Una de las cineritas registradas en la región de Salango-Río Blanco podría también, por su posición estratigráfica relativa, corresponder con este evento con la diferencia de que no parece haber causado un abandono de la zona o, si ha sucedido, éste ha sido de corta duración.

\section{APOGEO Y CATACLISMO (600-700 d. C.-1532)}

Concordamos con McEwan \& Delgado-Espinoza (2008) en notar que si efectivamente el término cacicazgo sigue vigente para ciertas áreas de la costa ecuatoriana, algunas poblaciones litorales ya han alcanzado un nivel de complejidad social mayor como en el caso de los manteños. Sin subrayar una vez más el control de las actividades mercantiles, las cuales deben sin embargo 
ser comprobadas efectivamente, algunos cambios que han afectado a la sociedad manteña (especialización, concentración poblacional, jerarquización marcada y adopción de una nueva arquitectura en ciertas regiones) sugieren este proceso de evolución sociocultural. Los grandes centros ubicados entre Manta y la desembocadura del río Portoviejo han desencadenado, con razón, un interés ininterrumpido desde los trabajos pioneros de Saville (1907-1910) y de Jijón y Caamaño (1997 [1952]) en la zona, seguidos luego por Estrada (1957). La considerable inversión, en tiempo y mano de obra, necesaria para la edificación del sinnúmero de realizaciones arquitectónicas (plataformas artificiales en Manta, tolas en Japoto o corrales en los Cerros de hojas y de Jaboncillos, así como en Chirije), atestiguan indirectamente del grado de control y organización de obras mayores como se puede averiguar en el recién excavado sitio de Japoto por el número de estructuras presentes (Bohórquez et al., 2003; Bouchard et al., 2006) y las elaboradas técnicas usadas (Bouchard, 2008). Al sur de la provincia de Manabí, otra entidad, el Señorío de Salangome, mencionado ya en las crónicas (Sámano, 1985 [1527]), alcanza un nivel de complejidad social sin duda igual aunque los vestigios monumentales se revelan menos variados. Una arquitectura rectangular, con edificios que alcanzan los $50 \mathrm{~m}$ de largo por $12 \mathrm{~m}$ de ancho, se encuentra con profusión en los sitios de Agua Blanca, varios centenares sobre una superficie de 4 km² (McEwan \& Delgado-Espinoza, 2008), y de Puerto López (Currie, 1995). Con la época Manteña, se alcanza el mayor nivel de densidad ocupacional con más de 100 sitios registrados en nuestra zona de estudio (fig. 3). Siguiendo un proceso iniciado durante el Desarrollo Regional, el valle del río Blanco muestra una dinámica muy intensa de ocupación concentrada al borde del cauce sobre las amplias terrazas aluviales, pero irradiando netamente en los valles adyacentes. Los alrededores de los actuales pueblos de Río Blanco y La Encantada, así como el valle bajo representan los dos focos de la ocupación territorial tierra adentro. Aldeas satélites se dispersan a lo largo de los pequeños tributarios, sobre las estrechas terrazas aluviales limitadas por las fuertes pendientes.

La cerámica de uso común presenta un grado real de estandardización. En todas las aldeas manteñas se han encontrado ollas de dimensión variada (hasta un diámetro de $44 \mathrm{~cm}$ ), con paredes a veces muy finas de aspecto externo rugoso y alisado en su interior (fig. $4 \mathrm{H}$ ). Otro artefacto recurrente, el cómale, aparece siempre en los conjuntos de esta época (fig. $4 \mathrm{I}$ ). Estas formas conforman respectivamente la urna y la tapa en los contextos funerarios como en el caso de la tumba con pozo y cámara lateral descubierta en el año 2003 en Salango (Graber \& Jastremski, 2009).

Los españoles, planificando conquista y dominio, trajeron consigo enfermedades cuyo impacto se hará evidente en las siguientes décadas con la exterminación ciega de la mayoría de la población prehispánica. Sin excluir una huida de ciertas comunidades a zonas inalcanzables por los europeos, el desarrollo de la cultura Manteña vivió un final abrupto por las pérdidas humanas sufridas y la organización colonial naciente. Según un documento anónimo de los primeros años del siglo XVII (Anónimo, 1994 [1604-1605]), la población de la bahía de Salango se resumía en un viejo cacique, sin descendencia, y a sus esposas: entonces 4 personas. La recuperación demográfica de la región ocurrirá lentamente a partir del inicio del siglo XX. 


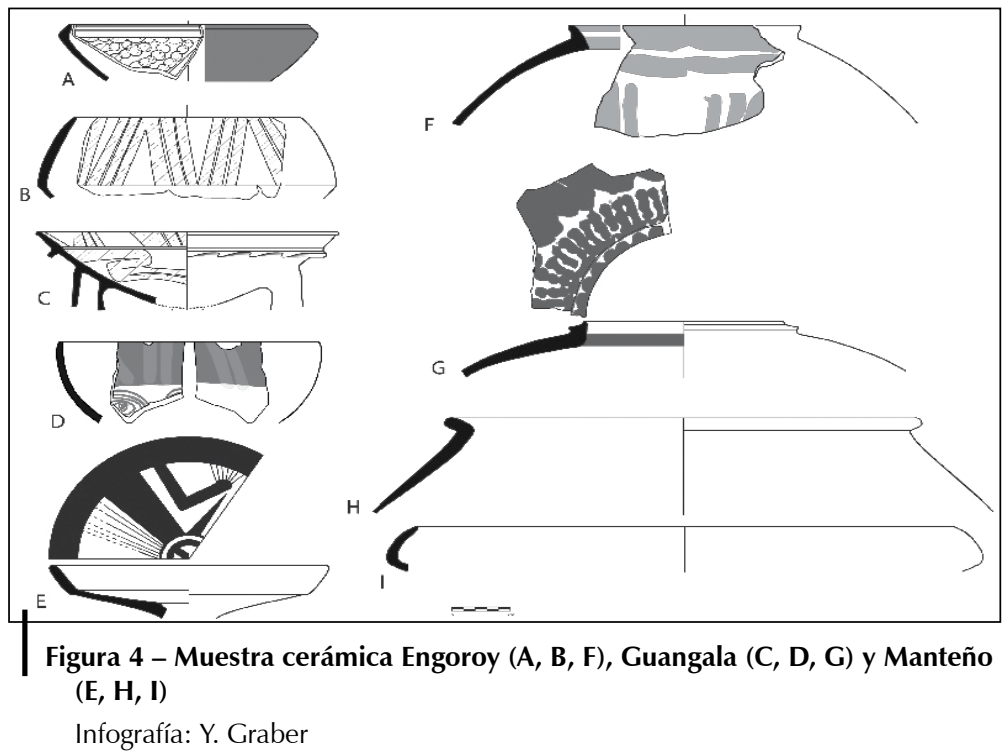

\section{CONCLUSIONES}

J. Damp (1984) destacó la sorprendente densidad de asentamientos Valdivia en el valle del río Blanco relacionada, por este autor, a las condiciones climáticas y medio ambientales que autorizaban la práctica de una agricultura en medio tropical húmedo. Fuertemente influenciado por las teorías de D. Lathrap, el valle del río Blanco servía para añadir una prueba más en contra del escenario difusionista de Meggers, Evans y Estrada. El sur de Manabí valía también de coartada para trasponer procesos socioculturales interpretados a partir de los vestigios de Real Alto en regiones ajenas. Si efectivamente existen recurrencias culturales, la arqueología debía comprobar, con la certeza a su alcance, la probabilidad de sus conclusiones en cada contexto regional. Efectivamente, las comunidades Valdivia conocieron un crecimiento cierto en el sur de Manabí, con toda la incertidumbre ligada a nuestros métodos de fechamiento actual para comprobar la contemporaneidad de los asentamientos, pero la filigrana social y ritual se nos escapaba todavía. A partir de las zonas más provechosas en término de espacio y recursos naturales explotables (la orilla oceánica y el amplio valle del río Blanco), pequeñas aldeas Valdivia alcanzaron paulatinamente áreas río arriba donde el espacio disponible no sostenía una población importante. Estrechamente ligadas, tal vez a través de lazos de parentesco, desarrollaron una red de intercambio local entre dos modos de aprovechamiento territorial distintos: uno estrechamente ligado a los recursos marinos y el otro dependiente del cultivo intensivo de las terrazas aluviales así como del aporte cinegético.

Si la disminución del número de sitios durante el Engoroy correspondió efectivamente a un fenómeno de aglutinación poblacional, entonces éste alcanzó su auge en los primeros siglos de nuestra era. La presión demográfica consecutiva 
inició un proceso de «expulsión» hacia las zonas marginales creando así una red de aldeas satélite resultantes de los asentamientos mayores. Sin embargo, pocos sitios Engoroy se ampliaran frente a las ocupaciones Machalilla anteriores fuera de ciertas excepciones como Puerto López, Salango o la zona de San Vicente. Queda entonces pendiente la cuestión de saber si algún o algunos fenómeno(s) natural(es) o proceso(s) social(es) indefinido(s) se unieron, sincrónicamente o no, para inducir una regresión demográfica contemporánea de la influencia creciente del recinto ceremonial de Salango. Su desaparición en el siglo VII d. C. evocaría cambios sociales profundos, culminación de un proceso obvio, aunque paulatino, de ampliación de los centros poblacionales principales a nivel regional y de una expansión de pequeñas aldeas secundarias que constituyeron uno de los componentes de la base económica de las entidades sociopolíticas en formación. La densificación de la red ocupacional culminó durante el periodo Manteño y se reveló particularmente marcada en el valle del río Blanco. Se sugirió que los asentamientos tierra adentro adquirieron importancia como proveedores de recursos, agrícolas entre otros, para sostener la clara especialización de ciertos sitios litorales en la producción de objetos de prestigios, como en el caso de los talleres de concha, evidenciados en Los Frailes, Salango y Río Chico, en este último con edificios de $50 \mathrm{~m}$ de largo.

\section{Agradecimientos}

Que las siguientes personas reciban aquí nuestros sinceros agradecimientos: Valentina Martínez, Mike Harris, Richard Lunniss, Patrick Gay, Felix Carvajal, Zaida Rodríguez, Jeremy Bleech, Michiel Dekker y todos los habitantes de las comunas de Salango, Pital y Las Tunas.

\section{Referencias citadas}

ANÓNIMO, 1994 [1604-1605] - Descripción de la ciudad de Guayaquil. In: Relaciones historico-geográficas de la Audiencia de Quito (siglo XVI-XIX), tome II (P. Ponce, ed.): 10-48; Quito, MARKA Instituto de Historia y Antropología Andina, Abya-Yala.

BINFORD, L. R., 1964 - A Consideration of Archaeological Research Design. American Antiquity, 29 (4): 425-441.

BOHORQUEZ, S., GRABER, Y., MEJÍA, F. \& SÁNCHEZ MOSQUERA, A., 2003 - Proyecto Japoto 2002; Guayaquil, ARKU y Ministerio de Turismo. Informe de Avances de la temporada 2002 entregado al INPC.

BOUCHARD, J.-F., 2008 - Japoto : une métropole régionale tardive de la province côtière du Manabí (Équateur). Les Nouvelles de l'archéologie, 111-112: 89-94.

BOUCHARD, J.-F., FUENTES, F. \& LÓPEZ, T., 2006 - Aldeas y pueblos prehispánicos en la costa de Manabí: Chirije y Japoto. Bulletin de I'Institut Français d'Études Andines, 35 (3): 243-256.

CURRIE, E., 1995 - Prehistory of the southern Manabí coast, Ecuador: López Viejo, iii + 64 рp.; Oxford: BAR International Series 618.

DAMP, J., 1984 - Environmental Variation, Agriculture, and Settlement Processes in Coastal Ecuador (3300-1500 B.C.). Current Anthropology, 25 (1): 106-111. 
ESTRADA, V. E., 1957 - Prehistoria de Manabí, 176 pp.; Guayaquil: Museo Victor Emilio Estrada.

GRABER, Y., 2008 - Aux origines du «Señorío de Salangome». Occupation territoriale préhispanique de la côte sud de Manabí (Équateur). Les Nouvelles de l'archéologie, 111-112: 59-64.

GRABER, Y. \& JASTREMSKI, N., 2009 - Étude d'une tombe collective de l'époque Manteño (Salango, Équateur) dans son contexte, culturel et funéraire, régional. Antropo, 18: 9-25. www.didac.ehu.es/antropo

GUILLAUME-GENTIL, N., 2008 - Cinq mille ans d'histoire au pied des volcans en Équateur; Golion: Infolio/SLSA/FNS (Terra Archeologica VI).

GUINEA, M., 1984 - Patrones de asentamiento en la arqueología de Esmeraldas (Ecuador), 243 pp.; Madrid: Ministerio de Asuntos Exteriores. Memorias de la Misión Arqueológica Española en el Ecuador 8.

HILL, B., 1972-1974 - A new chronology of the Valdivia ceramic complex from the coastal zone of Guayas province, Ecuador. Nawpa Paccha, 10-12: 1-32.

ISAACSON, J. S., 1994 - Sedimentos Volcánicos en Contextos Arqueológicos del Occidente del Ecuador. In: Regional Archaeology in Northern Manabí, Ecuador, I: Environment, Cultural Chronology, and Prehistoric Subsistence in the Jama River Valley (J. Zeidler \& D. M. Pearsall, eds): 132-140; Pittsburgh: Universidad de Pittsburgh. Memoirs in Latin American Archaeology 8.

JIJÓN Y CAAMAÑO, J., 1997 [1952] - Antropología prehispánica del Ecuador, 387 pp.; Quito: Museo Jacinto Jijón y Caamaño.

LIPPI, R. D., 1983 - La Ponga and the Machalilla Phase of Coastal Ecuador; Madison: Universidad de Wisconsin. Tesis de doctorado.

LUNNISS, R., 2001 - Archaeology at Salango, Ecuador: an Engoroy ceremonial site on the south coast of Manabí; Ann Harbor, UMI University Microfilms International.

LUNNISS, R., 2007 - Vida y costumbres de los pobladores del Ecuador antiguo, 149 pp.; Guayaquil: Museo Presley Norton.

LUNNISS, R., 2008 - Where the Land and The Ocean Meet : The Engoroy Phase Ceremonial Site at Salango, Ecuador, 600-100 BC. In: Pre-Columbian Landscapes of Creation and Origin (J. E. Staller, ed.); New York: Springer.

MARCOS, J., 1988 - Real Alto. La historia de un centro ceremonial Valdivia, 339 pp.; Guayaquil: ESPOL, Corporación Editora Nacional. Biblioteca Ecuatoriana de Arqueología 4.

MARTIN, A., 2009 - The Domestic Mode of Production and the Development of Sociopolitical Complexity: Evidence from the Spondylus Industry of Coastal Ecuador, 179 pp.; Pittsburg: Universidad de Pittsburgh. Tesis de doctorado.

MARTÍNEZ, V., GRABER, Y. \& HARRIS, M., 2006 - Estudios interdisciplinarios en la costa centro-sur de la provincial de Manabí (Ecuador): nuevos enfoques. Bulletin de I'Institut Français d'Études Andines, 35 (3): 433-444.

McEWAN, C., 2003 - «And the sun sits in his seats». Creating social order in Andean culture; Ann Harbor, UMI University Microfilms International.

McEWAN, C. \& DELGADO-ESPINOZA, F., 2008 - Late Pre-Hispanic Polities of Coastal Ecuador. In: Handbook of South American Archaeology (H. Silverman \& W. H. Isbell, eds.): 505-525; New York: Springer.

MESTER, A., 1990 - The pearl divers of Los Frailes: archaeological and ethnohistorical explorations of sumptuary goods trade and cosmology in the north and central Andes; Ann Harbor, UMI University Microfilms International. 
NORTON, P., LUNNISS, R. \& NAILING, N., 1983 - Excavaciones en Salango, provincial de Manabí, Ecuador. Miscelánea Antropológica Ecuatoriana, 3: 9-72.

PAULSEN, A. C. \& McDOUGLE, E. J., 1974 - The Machalilla and Engoroy Occupations of the Santa Elena Peninsula in South Coastal Ecuador; Washington: Society of American Archaeology, 39 Annual Meeting.

RAYMOND, J. S., 2003 - Social Formations in the Western Lowlands of Ecuador during the Early Formative. In: Archaeology of Formative Ecuador (J. S. Raymond \& R. L. Burger, eds.): 33-66; Washington D.C.: Dumbarton Oaks.

SÁMANO, J. de, 1985 [1527] - Relación Sámano. In: Francisco de Xérez. Verdadera relación de la conquista del Perú y la provincia del Cuzco (C. Bravo, ed.); Madrid: colección crónicas de América 14, Historia 16.

SAVILLE, M., 1907-1910 - The Antiquities of Manabí, vol. I y II, 135 pp. + 284 pp.; NewYork: Heye Museum:

SCHWARTZ, F. A. \& RAYMOND, J. S., 1996 - Formative settlement patterns in the Valdivia Valley, south-west coastal Ecuador. Journal of Field Archaeology, 23 (2): 205-224.

STALLER, J. E., 2001 - Reassessing the Developmental and Chronological Relationship of the Formative of Coastal Ecuador. Journal of World Prehistory, 15 (2): 193-256.

ZEIDLER, J. A., 1986 - La evolución local de asentamientos formativos en el litoral ecuatoriano: el caso de Real Alto. In: Arqueología de la Costa Ecuatoriana: Nuevos Enfoques (J. Marcos, ed.): 85-127; Quito: Corporación Editora Nacional.

ZEIDLER, J. A., 1995 - Archaeological survey and site discovery in the forested neotropics. In: Archaeology in the lowlands American tropics (P. W. Stahl, ed.): 7-41; Cambridge: Cambridge University Press.

ZEIDLER, J. A., 2003 - Settlement Process and Historical Contingency in the Western Ecuadorian Formative. In: Archaeology of Formative Ecuador (J. S. Raymond \& R. L. Burger, eds.): 69-123; Washington D.C.: Dumbarton Oaks.

ZEIDLER, J. A., 2008 - The Ecuadorian Formative. In: Handbook of South American Archaeology (H. Silverman \& W. H. Isbell, eds.): 459-488; New York: Springer.

ZEIDLER, J. A. \& PEARSALL, D. M., 1994 - Regional Archaeology in Northern Manabí, Ecuador, I: Environment, Cultural Chronology, and Prehistoric Subsistence in the Jama River Valley, 248 pp.; Pittsburgh: Universidad de Pittsburgh. Memoirs in Latin American Archaeology 8. 